\title{
Minority-Carrier Lifetime in InP as a Function of Light Bias
}

Jane A. Yater and I. Weinberg

National Aeronautics and Space Administration

Lewis Research Center

Cleveland, Ohio

and

Phillip P. Jenkins and Geoffrey A. Landis

NYMA, Inc.

Engineering Services Division

Brook Park, Ohio

Prepared for the

First World Conference on Photovoltaic Energy Conversion cosponsored by the IEEE, PVSEC-Japan, PVSEC-Europe

Waikoloa, Hawaii, December 5-9, 1994

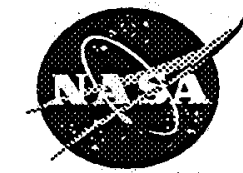

National Aeronautics and Space Administration
(WASA-TH-106021) MRORITY-CARRIER

LIFETIME IN INP AS A FUNCTION OF

LGHT BAS NASA. Lawis Research

$\operatorname{center} 60$
$N 95-13453$

Unelas 


\title{
MINORITY-CARRIER LIFETIME IN InP AS A FUNCTION OF LIGHT BIAS
}

\author{
Jane A. Yatert and I. Weinberg \\ NASA Lewis Research Center, Cleveland, OH 44135 \\ Phillip P. Jenkins and Geoffrey $A$. Landis \\ NYMA, Inc., Brookpark, OH 44142
}

\begin{abstract}
Minority-carrier lifetime in $\ln P$ is studied as a function of doping level and laser intensity using time-resolved photoluminescence. A continuous wave diode laser illuminates bulk InP and acts as a light bias, injecting a steady-state concentration of carriers. A 200 ps laser pulse produces a small transient signal on top of the steady-state luminescence, allowing lifetime to be measured directly as a function of incident intensity. For $\mathrm{p}-\ln \mathrm{P}$, lifetime increases with light bias up to a maximum value. Bulk recombination centers are presumably filled to saturation, allowing minority carriers to live longer. The saturation bias scales with dopant concentration for a particular dopant species. As light bias is increased for $n-\ln P$, minority-carrier lifetime increases slightly but then decreases, suggesting radiative recombination as a dominant decay mechanism.
\end{abstract}

\section{INTRODUCTION}

Measurement of minority-carrier lifetime $(\tau)$ is important for the design of photovoltaic devices. $\ln P$ is a promising semiconductor for space solar cells but relatively few measurements of its minority-carrier properties have been reported [1]. Measured efficiencies of $\ln P \mathrm{n} / \mathrm{p}$ solar cells are significantly lower than expected from theoretical models [2], suggesting that the materials properties deteriorate during processing. A better understanding of the mechanisms controlling $\tau$ in $\ln P$ is needed.

Minority-carrier lifetime is influenced by radiative and non-radiative recombination mechanisms $[3,4]$. A separate lifetime is associated with each mechanism, and $\tau_{\text {total }}$ is calculated as the inverse sum of the reciprocal lifetimes. The radiative lifetime is expressed as

$$
\tau_{\mathrm{R}}=\frac{\phi}{B(N+\Delta n)}
$$

where $\mathrm{B}$ is the radiative recombination coefficient, $\Delta n$ the excess carrier concentration, and $\mathbf{N}$ the doping level. $\phi$ is the photon recycling factor (self-absorption and reemission of light), which can greatly enhance $\tau_{R}$ [5]. For defect-free, direct bandgap material, the lifetime may be limited by radiative recombination.

Cell efficiency can be adversely affected by chemical and structural defects such as impurity atoms and

† National Research Council-NASA Research Associate at Lewis Research Center. dislocations, which create energy levels in the forbidden gap and increase non-radiative, or Shockley-ReadHall (SRH), recombination. Free surfaces create surface states which act similarly. SRH lifetime is expressed as [6]

$$
\tau_{\mathrm{SRH}}=\frac{\tau_{\min }(N+\Delta n)+\tau_{\mathrm{maj}} \Delta n}{N+\Delta n}
$$

where $\tau_{\min }$ and $\tau_{\operatorname{maj}}$ are the minority and majority-carrier lifetimes. As plotted in Fig. 1, $\tau_{\text {SRH }}$ rises with carrier concentration as recombination centers are filled and plateaus at $\tau_{\min }+\boldsymbol{\tau}_{\mathrm{maj}}$. $\boldsymbol{\tau}_{\mathbf{R}}$ drops rapidly from the low injection value $(\Delta n \ll N)$ as the carrier concentration surpasses the doping.

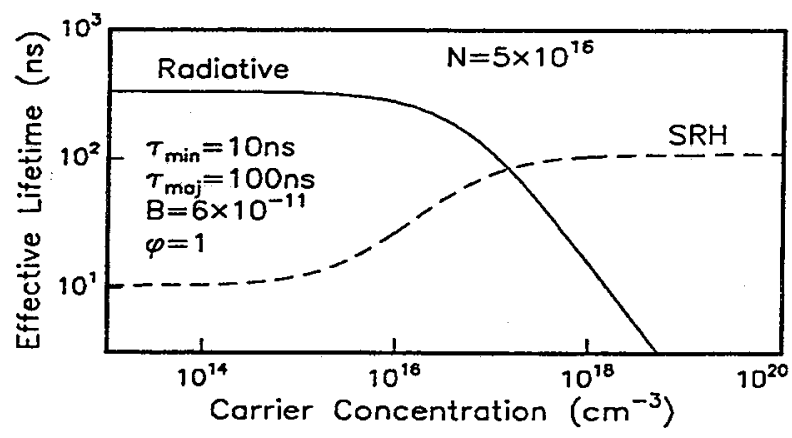

Fig. 1. Calculated radiative and SRH lifetimes.

For direct bandgap semiconductors such as $\ln P$, the most common technique for determining minority-carrier lifetime is time-resolved photoluminescence (PL). Following excitation of the material with a short laser pulse, the transient $\mathrm{PL}$ decay is recorded. At low injection, the signal should follow a single exponential decay. However, lifetime varies with carrier concentration, thus complicating evaluation of the transient signal at higher injection $(\Delta n \geq N)$ [3]. In this work, a unique experimental design simplifies the often complex interpretation of transient luminescence by probing $\tau$ at specific levels of carrier concentration. Data for n-type and p-type InP are presented as a function of doping level and light bias.

\section{EXPERIMENTAL PROCEDURE}

Bulk InP wafers were grown by Nippon Mining using liquid-encapsulated Czochralski growth. P-type wafers are doped with $\mathrm{Zn}$ and $\mathrm{Cd}$ from $1.8 \times 10^{16}$ to $9 \times 10^{18} \mathrm{~cm}^{-3}$ and n-type $\ln P$ is doped from $3 \times 10^{16}$ to $9 \times 10^{18} \mathrm{~cm}^{-3}$ 


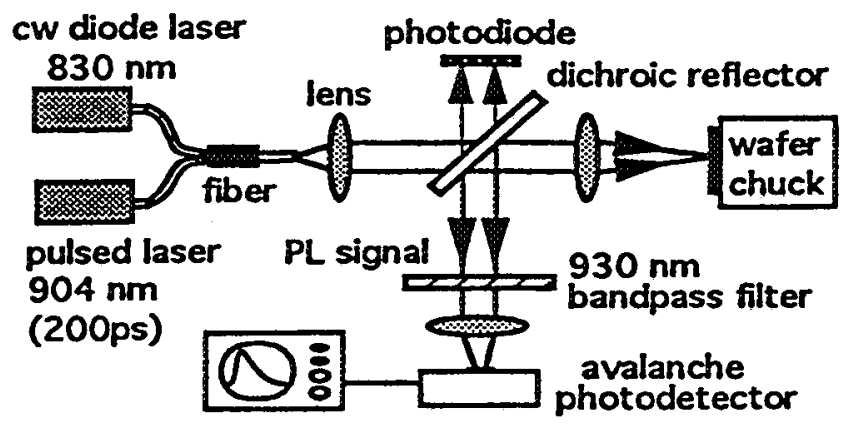

Fig. 2. Experimental apparatus.

with $\mathrm{S}$ and $\mathrm{Sn}$. No special surface preparation was done to the wafers, with lifetime measurements acquired over 12 months showing no significant change. The experimental apparatus, depicted in Fig. 2, has been described previously [7]. Continuous wave (CW) diode laser light at $830 \mathrm{~nm}$ passes through an optical fiber multiplexer before being collimated and focused to a $50 \mu \mathrm{m}$ spot. This cw illumination acts as a light "bias", injecting a steady state concentration of carriers.

The InP wafer is also excited with $35 \mathrm{kHz}, 200$ ps pulses of $904 \mathrm{~nm}$ diode laser light, which produce a small transient signal on top of the steady state luminescence, allowing lifetime to be measured directly as a function of CW laser intensity. The excitation pulses follow the same optical path as the $\mathrm{CW}$ light after entering the 50 $\mu \mathrm{m}$ diameter " $\gamma$ " fiber. A reflected portion of the laser light is measured by a calibrated photodiode to monitor the incident intensity. Light bias readings are accurate to within $\pm 10 \%$, accounting for small drifts in the diode laser output as well as calibration errors.

The InP luminescence is separated from reflected laser light by a dichroic reflector and narrow bandpass filters. The PL signal is detected by a Si avalanche photodetector and saved by a $20 \mathrm{GHz}$ digital sampling oscilloscope. Computer-controlled averaging over thousands of pulses improves the signal to noise ratio. The decay is fit to a theoretical expression assuming a single lifetime, $\tau$, and high surface recombination velocity (SRV $\geq 10^{6}$ $\mathrm{cm} / \mathrm{s}$ ) [8]. The fit is accurate to $\pm 5 \%$. Fig. 3 shows $P L$ decay curves corresponding to three levels of CW light bias for $\ln P: \mathrm{Zn}$ at $2.6 \times 10^{16} \mathrm{~cm}^{-3}$. The fit to each set of data uses the value of $\tau$ shown in the figure. In general, the experimental apparatus is easy to use and allows for fairly rapid throughput of samples.

\section{LIFETIME RESULTS FOR P-InP}

Minority carrier lifetime is significantly shorter in pInP than $n-\ln P$ at comparable doping levels. With no light bias, the measured lifetime varies from around 10 ns to under $0.5 \mathrm{~ns}$ (the limit of the system resolution) for p-doping between $1.8 \times 10^{16}$ and $4 \times 10^{18} \mathrm{~cm}^{-3}$. As CW light bias is added, the lifetime increases up to a maximum value, as shown in Figs. $4 a$ and $4 b$ for $\mathrm{Zn-}$ and $\mathrm{Cd}$-doped InP. SRH sites are presumably filled to saturation, allowing minority carriers to live longer. The saturation value represents a 6-fold increase over the zero-bias lifetime for the four samples exhibiting light bias effects.

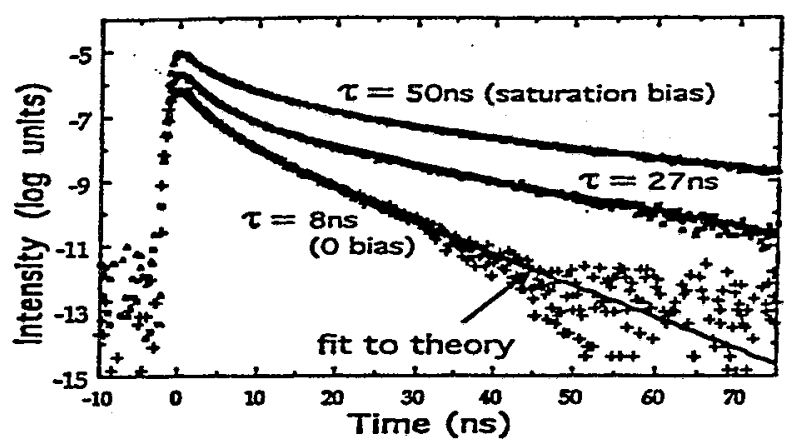

Fig. 3. PL decay from $p-\operatorname{In} P$ at 3 levels of $C W$ bias.

Even at saturation, however, $\tau$ remains significantly below the low-injection radiative limit. The increase in minority-carrier lifetime is insignificant until high levels of light bias are reached. The lowest doped sample shows the most dramatic increase, a 4-fold improvement at approximately 500 suns. Thus, for most solar cell applications, zero-bias lifetimes are appropriate, although samples doped below $1 \times 10^{18} \mathrm{~cm}^{-3}$ may demonstrate lifetime enhancement at significantly lower bias levels.
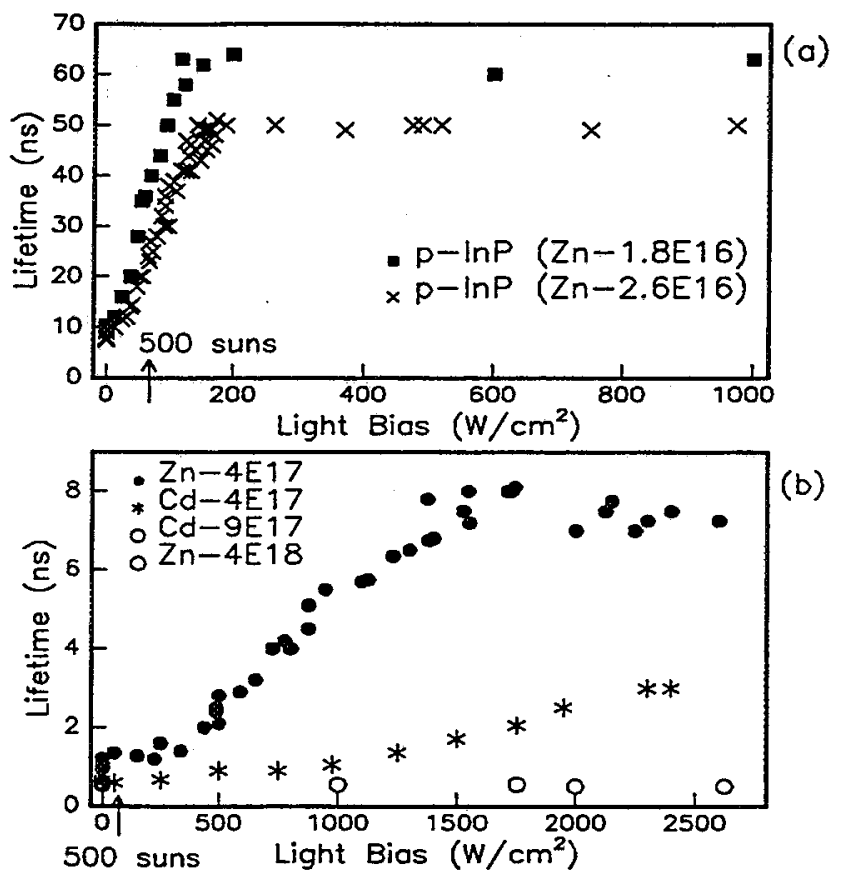

Fig. 4. Effective minority carrier lifetime as a function of light bias for $p$-InP. Doping is indicated.

Minority-carrier lifetime varies not only with doping level but also with dopant element. In Fig. 4b, InP doped to $4 \times 10^{17} \mathrm{~cm}^{-3}$ with both $\mathrm{Zn}$ and $\mathrm{Cd}$ show different behavior. InP:Zn has a longer lifetime and reaches SRH saturation at lower light bias. In comparing only Zndoped samples, the saturation value of light bias scales roughly with the doping level, implying that the number of SRH sites also scales with doping. Hence, the defect 
site is directly related to the dopant species. Since $\mathrm{Cd}$ suppresses the lifetime more than $\mathrm{Zn}$, it may create defect states closer to mid-gap which are more effective electron recombination sites. These results may indicate that $\mathrm{Zn}$ is preferable to $\mathrm{Cd}$ for doping InP solar cells.

$\mathrm{Cd}$-doped $\ln \mathrm{P}$ also appears to contain light-saturable defect sites not evident in the $\mathrm{Zn}$-wafers. During data acquisition at high light bias, the total PL signal slowly increases with time. After blocking the beam for several minutes, the PL intensity has fallen towards its initial value, but immediately begins to rise again. A slight increase in lifetime often accompanies these events.

Analysis of $\mathrm{p}-\ln \mathrm{P}$ doped higher than $4 \times 10^{17} \mathrm{~cm}^{-3}$ is limited by the system resolution, since measured lifetimes are consistantly below 0.5 ns. As the density of defect sites increases, the light bias is not sufficient to even partially saturate the recombination centers and increase measured lifetimes. In addition, the radiative limit of highly doped InP falls to a few ns, meaning that light bias will never enhance $\tau$ beyond that level.

For SRH-dominated recombination, the saturation value of lifetime equals the sum of $\tau_{\min }$ and $\tau_{\text {maj }}$ [5], while the zero-bias (low injection) value of lifetime is $\tau_{\text {min }}$. Fig. 5 shows a plot of $\tau_{\text {min }}$ and $\tau_{\text {maj }}$ as derived from the data. The data are consistant with previous results [7]. The ratio of majority to minority-carrier lifetime varies from 4 to 6.5 for the samples tested.

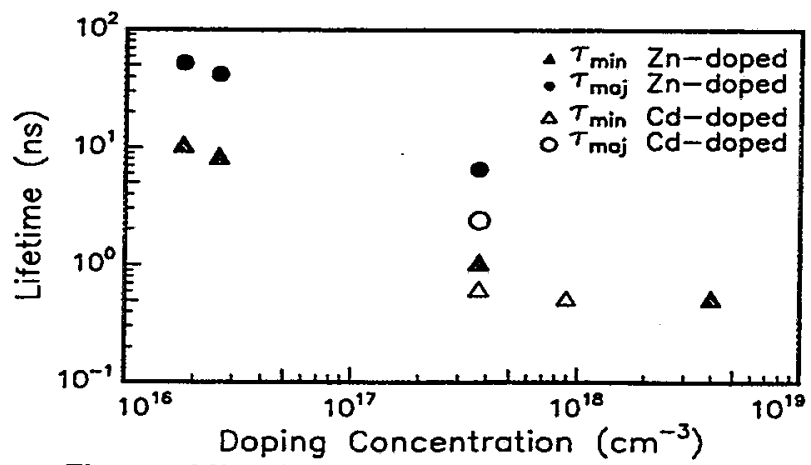

Fig. 5. Minority and majority lifetime for $p-\ln P$.

\section{LIFETIME RESULTS FOR N-InP}

N-type InP lifetimes are significantly longer than those observed in p-type InP, as seen in Figs. $6 a$ and $6 \mathrm{~b}$. Fig. 6a shows data from three lightly- to moderatelydoped samples. The zero-bias, low injection lifetime varies from 200 to $250 \mathrm{~ns}$, but an abrupt rise in $\tau$ occurs as light bias is added to the excitation pulse. Lifetime values begin to fall above $50-100 \mathrm{~W} / \mathrm{cm}^{2}$ for all samples. Highly doped wafers exhibit less sensitivity to light bias, as is evident in Fig. 6b. The Sn-doped InP cannot be directly compared to InP:S, but its behavior is similar.

The measured zero-bias lifetime lies above or near the radiative limit for these $n$-InP samples, suggesting that the limiting minority-carrier decay mechanism is radiative rather than $\mathrm{SRH}$ recombination. The decrease in lifetime with increasing light bias further supports such a premise since radiative lifetime goes as $1 / \mathrm{B}(N+\Delta n)$. Photon recycling, previously identified as a contributing factor to long lifetimes [5], also appears to influence these

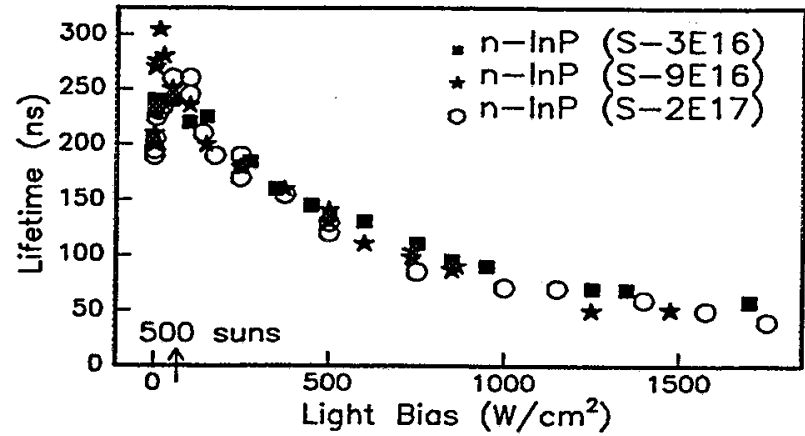

(a)

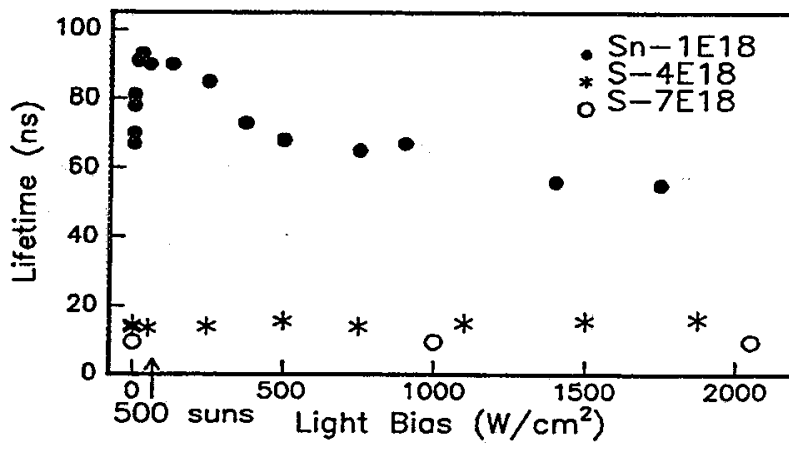

(b)

Fig. 6. Effective minority carrier lifetime as a function of light bias for $n-\ln P$. Doping is indicated.

results. A comparison of the data (both zero-bias and maximum observed values) and the low injection radiative lifetime is shown in Fig. 7. Assuming the $\ln P B$ coefficient equals the GaAs value of $2 \times 10^{-10} \mathrm{~cm}^{3} / \mathrm{s}$, every sample is indeed above the radiative limit. $A$ recent experiment determined that, for $\ln P, B \geq 6 \times 10^{-11} \mathrm{~cm}^{3} / \mathrm{s}$ [9], thus raising $\tau_{R}$ by a factor of three. Again, all but the lowest doped wafer still exceed the radiative limit. A fit of the three data points from the highest doped samples, shown in Fig. 7 , gives a slope essentially parallel to the radiative lifetime. The offset indicates a photon recycling factor of $\phi=4.2$ (or $\phi=14$ for $B=2 \times 10^{-10} \mathrm{~cm}^{3} / \mathrm{s}$ ). This represents a lower bound on $\phi$ since other recombination mechanisms may exist which lower the lifetime.

While radiative recombination appears to be a primary mechanism for decay of holes in $n-I n P$, SRH recombination also influences the decay process. in Fig. $6 a$, measured lifetimes for the three InP:S wafers follow essentially the same curve with increasing light bias, even though their doping varies by an order of magnitude. Likewise, the relatively flat trend of the data below $10^{18} \mathrm{~cm}^{-3}$ in Fig. 7 suggests that SRH recombination limits $\tau$ in this regime, since radiative lifetimes scale with doping. These experimental results are consistant with other studies $[7,8,10]$, where moderately doped $n-\ln P$ lifetimes lie around $300 \mathrm{~ns}$ with scatter of $\pm 100 \mathrm{~ns}$.

The most telling evidence of SRH influence on lifetime is the initial increase in $\tau$ with light bias. Unlike p-InP (where lifetime saturates at lower values and at higher levels of light bias with increased doping), the lowbias data shows scatter and no obvious doping dependence. The interplay between radiative recombination and non-radiative decay processes may be more complex in $n-\operatorname{InP}$. While the nature of the SRH site is not 


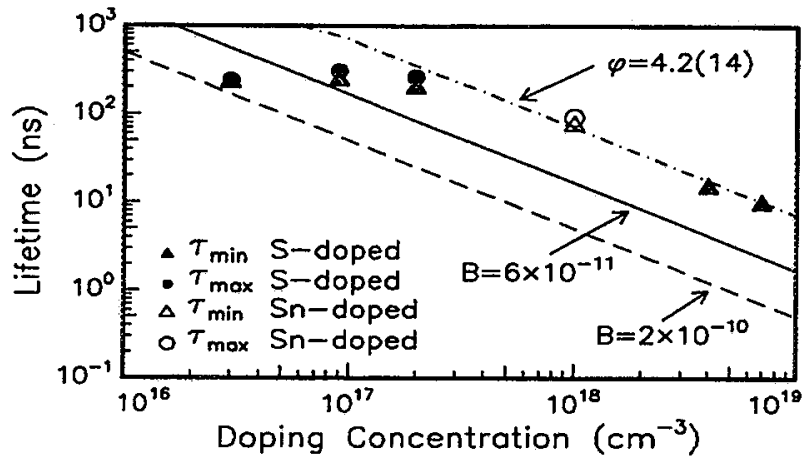

Fig. 7. Minority-carrier lifetime for $n-\ln P$ with no light bias. Lines are radiative lifetimes.

known, saturation of surface states is probably not the cause of the initial rise in lifetime. The theoretical fit to the experimental data is performed away from the initial, surface-dependent decay transient, making the measured lifetimes relatively insensitive to surface effects.

For planar solar cells, the zero-bias lifetime is again appropriate. Concentrator cells may be affected by the increase in lifetime observed over the first few hundred suns, and perhaps even by the subsequent decrease. However, lifetime in processed cells may behave quite differently, as photon recycling may vary and non-radiative recombination sites may be introduced.

\section{DISCUSSION}

Minority-carrier lifetime varies with light bias differently for $n-\ln P$ and $p-\ln P$. To consider the influence of various recombination mechanisms on the minoritycarrier decay, the light bias must be converted to carrier concentration and the experimental data compared to theoretical calculations of lifetime. While space is insufficient to fully explore such an analysis, a brief description of the calculations will be given.

The CW laser illumination creates electron-hole pairs near the surface, which diffuse into the substrate. The steady state concentration of carriers is determined by solving the diffusion equation using known or estimated values of incident flux, absorption coefficient, ambipolar diffusion length (L) and diffusion coefficient (D), and SRV [11]. Using the experimental value of $\tau, L$ is calculated as $\sqrt{\mathrm{D} \tau}$. Results are highly dependent on the chosen value of SRV. Because the steady state profile is non-uniform, the average value of carrier concentration within one absorption depth of the surface is used.

The expression for minority-carrier lifetime is

$$
1 / \tau_{\text {total }}=1 / \tau_{\text {SRH }}+1 / \tau_{R}+1 / \tau_{\text {surf }}+1 / \tau_{\text {Auger }},
$$

where the individual lifetimes correspond to SRH, radiative, surface and Auger recombination, respectively. Auger recombination does not dominate until very high injection, and the surface term is unnecessary for this work since bulk recombination lifetimes are determined experimentally. Thus, $\tau_{\text {total }}$ can be calculated as a function of carrier concentration using eqns. (1) and (2).

The rise and fall of the $p-\ln P$ and $n-\ln P$ data, respectively, can be fit reasonably well by varying several parameters. However, for $\mathrm{p}-\mathrm{InP}$, the lifetime data as a function of bias intensity shows a "plateau", where $\tau$ reaches a broad maximum. Calculated lifetime, on the other hand, shows a decrease at higher intensities unless $\phi$ is increased to rather unphysical values of 200-500, suggesting that more complex analysis is required. Further details of the calculations will be given elsewhere.

\section{CONCLUSIONS}

Minority-carrier lifetime in InP has been studied as a function of doping level and laser intensity using timeresolved $P L$ with an additional $C W$ light bias. For $p-\operatorname{In} P$, $\tau$ increases with light bias up to a maximum value, characteristic of $\mathrm{SRH}$ recombination. The saturation bias scales with dopant concentration for a particular dopant species, indicating that $\mathrm{SRH}$ sites are directly related to the dopant atoms. For $n-\ln P, \tau$ increases slightly with light bias but then decreases, characteristic of radiative recombination. However, SRH decay mechanisms also play a role in $n-\ln P$, as the initial increase in $\tau$ suggests. Comparison of experimental data with calculated lifetimes must be pursued to better understand the mechanisms controlling minority-carrier lifetime in $\ln P$.

\section{REFERENCES}

[1] R.K. Ahrenkiel et al., "Recombination Lifetime and Performance of III-V Compound Photovoltaic Devices", Twenty Third IEEE PVSC, 1992, p. 42.

[2] M.B. Spitzer, B. Dingle, and R. Morrison, "A Review of InP Space Solar Cell Fabrication Technology" , Twenty First IEEE PVSC, 1990, p. 196.

[3] D.C. Marvin, S.C. Moss, and L.F. Halle, "Analysis of Transient PL Measurements on GaAs and AlGaAs Double Heterostructures", J. Appl. Phys., 72, 1992, p. 1970.

[4] R.K. Ahrenkiel, Semiconductors and Semimetals, Vol. 39, (Academic Press, Boston, 1993). Chapter 2.

[5] R.K. Ahrenkiel, B.M. Keyes, and D.J. Dunlavy, "Nonlinear Recombination Processes in Photovoltaic Semiconductors", Solar Cells, 30, 1991, p. 163.

[6] W. Shockley and W.T. Read, Jr., Phys. Rev., 87, 1952 , p. 835.

[7] G.A. Landis, P. Jenkins, and I. Weinberg, "Photoluminescence Lifetime Measurements in InP Wafers", Third IEEE International Conf. on InP and Related Compounds, 1991, p. 636.

[8] P. Jenkins et al., "Minority Carrier Lifetime in InP", Twenty Second IEEE PVSC, 1991, pp. 177-181.

[9] B.M. Keyes et al., "Time-resolved Photoluminescence of Undoped In P", J. Appl. Phys., 75, 1994, p. 4249.

[10] Y. Rosenwaks, Y. Shapira, and D. Huppert, "Picosecond Time-Resolved Luminescence Studies of Surface and Bulk Recombination Processes in InP", Phys. Rev. B, 45, 1992, p. 9108.

[11] H.B. Bebb and E.W. Williams, Semiconductors and Sermimetals, Vol. 8, (Academic Press, NY, 1972).Ch. 4. 


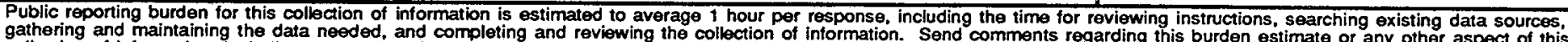

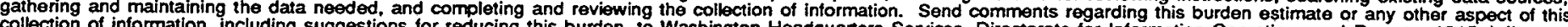

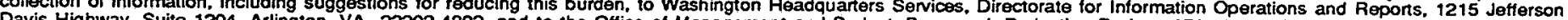
Davis Highway, Suite 1204, Arlington, VA 22202-4302, and to the Office of Management and Budget, Paperwork Reduction Project (0704-0188), Washington, DC 20503.

\begin{tabular}{l|l} 
1. AGENCY USE ONLY (Leave blank) & 2. REPORT DATE
\end{tabular}

4. TITLE AND SUBTITLE

January 1995

3. REPORT TYPE AND DATES COVERED

Technical Memorandum

Minority-Carrier Lifetime in InP as a Function of Light Bias 5. FUNDING NUMBERS

6. AUTHOR(S)

Jane A. Yater, I. Weinberg, Phillip P. Jenkins, and Geoffrey A. Landis

WU-233-01-OA

7. PERFORMING ORGANIZATION NAME(S) AND ADDRESS(ES)

National Aeronautics and Space Administration

Lewis Research Center

Cleveland, Ohio 44135-3191

8. PERFORMING ORGANIZATION REPORT NUMBER

E-9363

9. SPONSORING/MONITORING AGENCY NAME(S) AND ADDRESS(ES)

10. SPONSORINGMONITORING AGENCY REPORT NUMBER

National Aeronautics and Space Administration

Washington, D.C. 20546-0001

NASA TM-106821

11. SUPPLEMENTARY NOTES

Prepared for the First World Conference on Photovoltaic Energy Conversion cosponsored by the IEEE, PVSEC-Japan and PVSEC-Europe, Waikoloa,

Hawaji, December 5-9, 1994. Jane A. Yater, National Research Council-NASA Research Associate at Lewis Research Center, I. Weinberg, NASA

Lewis Research Center, Phillip P. Jenkins and Geoffrey A. Landis, NYMA Inc, Engineering Services Division, 2001 Aerospace Parkway, Brook Park, Ohio 44142. Responsible person, Jane A. Yater, organization code 5410, (216) 433-8485.

12a. DISTRIBUTION/AVAILABILTTY STATEMENT

Unclassified -Unlimited

Subject Category 76

This publication is available from the NASA Center for Aerospace Information, (301) 621-0390.

13. ABSTRACT (Maximum 200 words)

Minority-carrier lifetime in InP is studied as a function of doping level and laser intensity using time-resolved photoluminescence. A continuous wave diode laser illuminates bulk InP and acts as a light bias, injecting a steady-state concentration of carriers. A 200 ps laser pulse produces a small transient signal on top of the steady-state luminescence, allowing lifetime to be measured directly as a function of incident intensity. For p-InP, lifetime increases with light bias up to a maximum value. Bulk recombination centers are presumably filled to saturation, allowing minority carriers to live longer. The saturation bias scales with dopant concentration for a particular dopant species. As light bias is increased for n-InP, minority-carrier lifetime increases slightly but then decreases, suggesting radiative recombination as a dominant decay mechanism.

14. SUBJECT TERMS

Minority carrier lifetime; Indium phosphide; Photoluminescence

17. SECURTYY CLASSIFICATION OF REPORT Unclassified
18. SECURITY CLASSIFICATION OF THIS PAGE Unclassified
19. SECURITY CLASSIFICATION OF ABSTRACT Unclassified 